\title{
Reconstructing David Huffman's Legacy in Curved-Crease Folding
}

\section{Erik D. Demaine, Martin L. Demaine and Duks Koschitz}

David Huffman's curved-crease models are elegant, beautiful, and illustrative of Huffman's fascination with curved creases. Huffman's death in 1999 left us without his deep understanding, but his many models and notes provide a glimpse into his thinking. This paper presents reconstructions of some of David Huffman's curved crease patterns and models, aiming to recover his insight and uncover the mathematical beauty underlying the artistic beauty. These initial reconstructions represent the beginning of an ongoing project with the Huffman family to study and document David Huffman's work in folding.

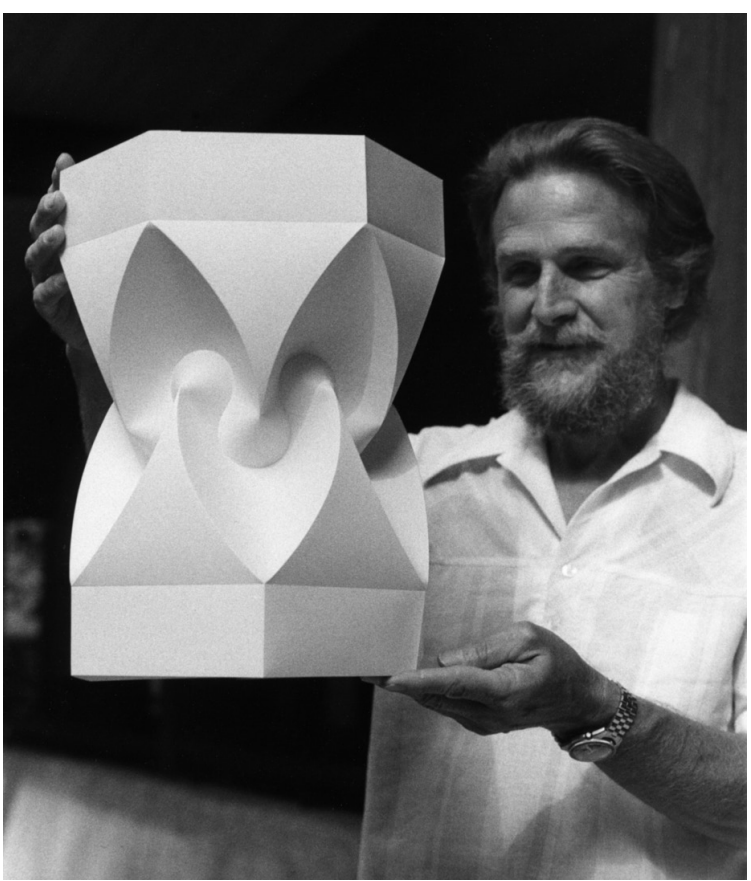

Figure 1. David Huffman and his "hexagonal column with cusps". (photo courtesy of UC Santa Cruz) 
The first known reference of curved-crease folding is the work of a Bauhaus student in a course by Josef Albers in 1927-1928 [Wingler 69]. This model has creases in concentric circles and a hole in the center. Since the 1930s, Irene Schawinsky, Thoki Yenn and Kunihiko Kasahara have built similar models with variations on the pleats and the size of the hole [Demaine et al 08]. Other intricate curved-crease origami sculpture was designed by Ronald Resch in the 1970s. From the 1970s to the 1990s, Huffman created hundreds of models that represent the majority of work done in this field [Wertheim 04]. He inspired further work on curved creases [Fuchs et al. 99] and research on finding the nearest proper folding that approximates a 3D scanned physical model [Kilian et al 08].

David Huffman was simultaneously studying the math and the art of curvedcrease origami. He analyzed the local mathematical behavior of curved creases in his Curvature and creases: A primer on paper [Huffman 76] and made sculptures to further study this special kind of folding. Our goal is to better understand the behavior of curved creases demonstrated in his models, given the lack of mathematical and algorithmic tools for designing curved-crease origami.

\section{Approach}

We are experimenting with both physical models and computer models to reconstruct Huffman's work. We analyze Huffman's designs by carefully studying photographs, measuring his models and studying features that occur frequently in his designs. Based on personal communication with David Huffman in 1998, we assume that almost all of Huffman's creases are conic section (quadratic) curves. His work spans across many areas of mathematical origami and here we select a few designs that use several quadratic curves within a single design or combine curved and straight creases.

\subsection{Folding methods}

The Huffman family provided us with an opportunity to see Huffman's estate, which they manage, and to see a variety of working models and crease patterns. We studied his techniques and work methods by looking at his drawing tools, templates and model-making equipment. Huffman transferred his crease patterns onto sheets of white, matt PVC ("vinyl") that is 0.01 " thick using French curves. He then traced the creases with a spring-loaded ball burnisher similar to the one shown in Figure 2 to precrease the material. He slowly bent the material to the maximal angle without kinking the uncreased material. The careful and timeconsuming folding technique attests to his incredible patience and love for craft when producing his art. 


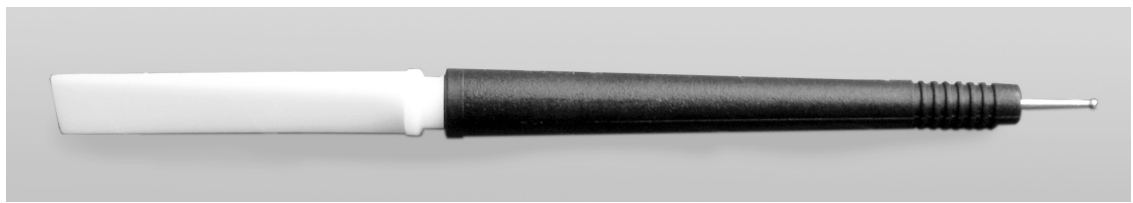

Figure 2. Huffman used a spring-loaded ball burnisher similar to this one.

\subsection{Reconstruction methods}

As part of this reconstruction effort we have decided to stay close to Huffman's way of making models. We deviate from his manual drawing methods in order to create digital files of the crease patterns. The reconstructions are drawn with CAD software, Rhinoceros 3D, which allows the use of quadratic curves. Most designs are drawn in 2D, folded and then visually analyzed. Some designs have been recreated virtually in 3D and then made into physical models. We make paper versions before producing the final versions from the exact same material Huffman used in the 70s.

We use an industrial vinyl cutter/printer to precrease the patterns, which require format translation to Adobe Illustrator. The $i$-Cut $i$-XL-24M Flatbed Cutter and Router has a 65 " by 120 " vacuum plate and 1 " cutting depth. The machine is furnished with 2 heads and can crease and cut at the same time. The drop-in tool slots allow the use of a special tool called a creasing wheel that gently pushes down onto a surface. The precreased vinyl then needs to be folded into its final shape by hand. Most of the reconstructions are close to the size of the originals. We decided to enlarge some examples to ensure better results, as tolerances become less of an issue.

\section{Reconstructions}

Huffman created a wide variety of models, most of which were never shown. The reconstructions selected for this paper are grouped into categories that highlight some of the observations we have made during our study. Of course aesthetic qualities and beauty are a big factor and it is impossible to know Huffman's exact motivations for each design.

We believe that David Huffman was interested in studying vertices of various degrees and that he made some of his models to show how they can be used. He studied vertices that are exploded and separated by polygons. Tessellations represent a large portion of his work, but we only include some that use curved creases. He further studied foldings that have the characteristic of describing a volume. The crease patterns we show in Figures 3 through 14 have tags that identify circles, ellipses and parabolas (see: ci, el, pa). 


\subsection{Degree-1 and -2 vertices}

The model in Figure 3 shows two mountain creases ending within the area of the paper. These vertices are located on the major axis of the ellipse of the valley crease, and the rulings around each degree- 1 vertex describe a cone surface. The valley crease is drawn by splicing ellipses together. This design was published on the website Graphica Obscura [Haeberli 96].
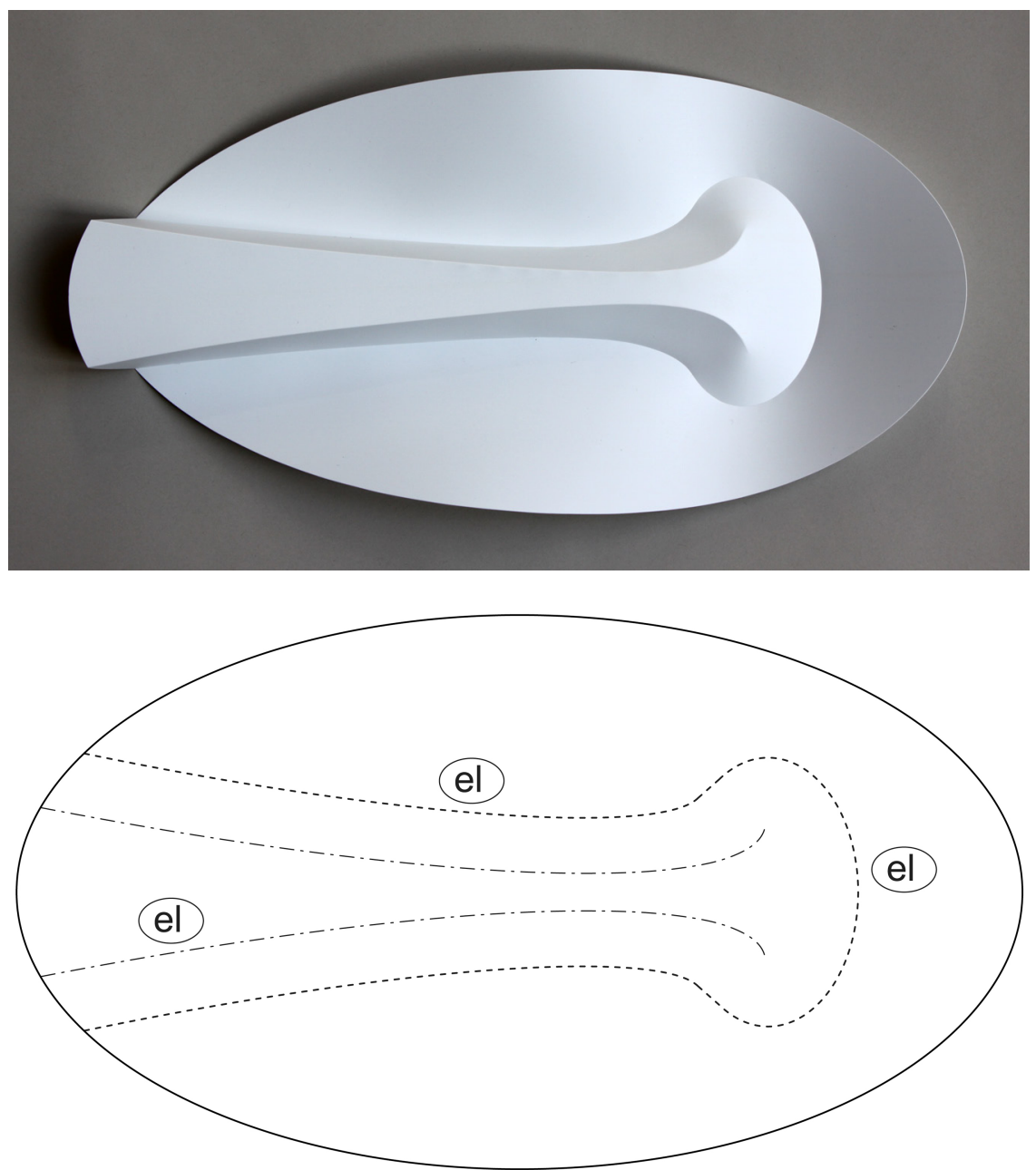

Figure 3. Huffman design using ellipses with two degree-1 vertices. 
The model shown in Figure 4 [Haeberli 96] uses two degree-2 vertices and is drawn using ellipses. There is a ruling line between the two points, but no crease is necessary. The cut-out shape is an ellipse and the mountain and valley assignments alternate radially.
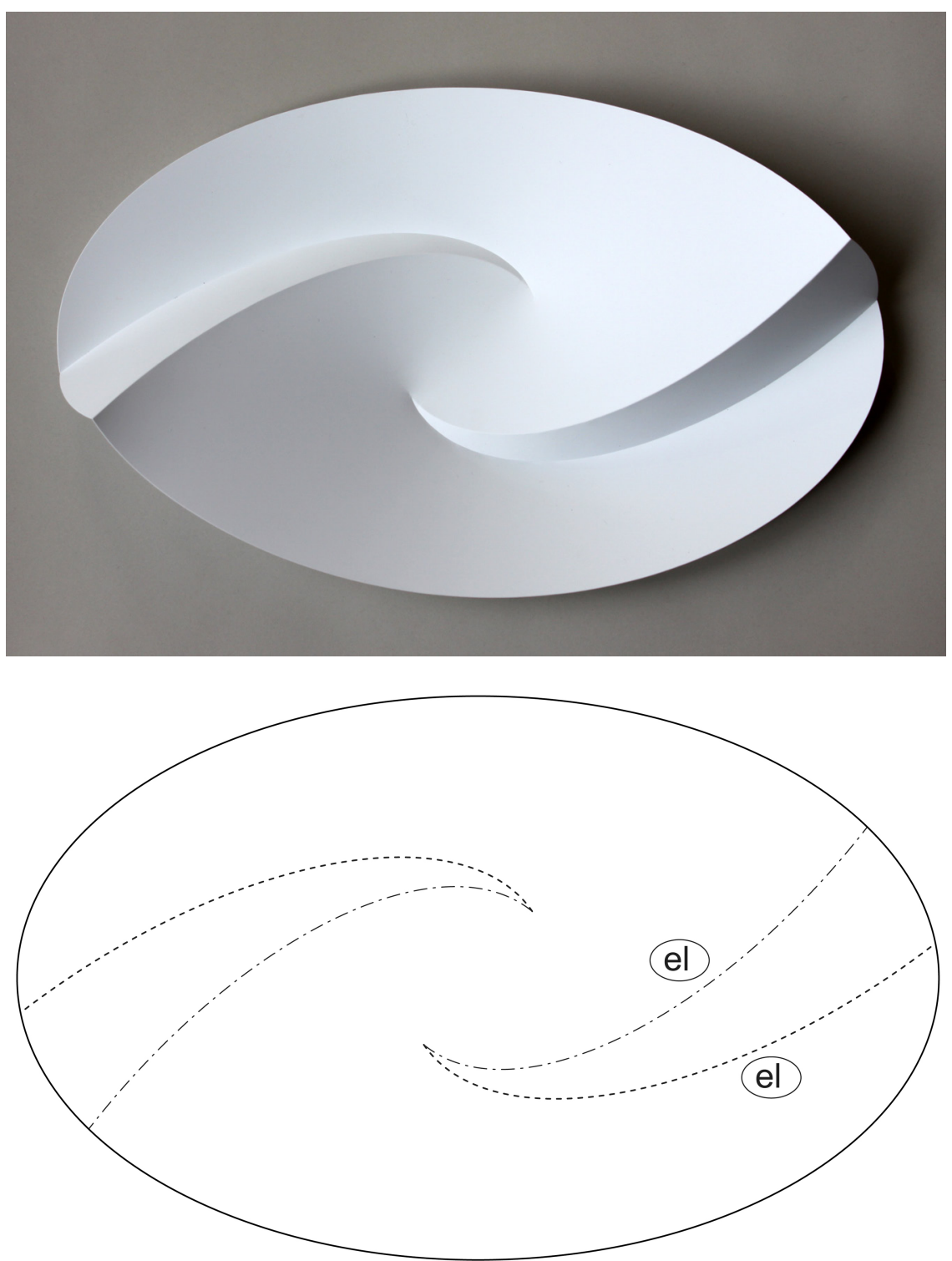

Figure 4. Huffman design using ellipses with two degree-2 vertices. 


\subsection{Inflated vertices}

We observe that Huffman was studying crease patterns with "exploded" vertices of varying degrees. The next series of models shows vertices that are exploded or inflated into flat polygons. Figure 5 shows a non-inflated degree- 4 vertex with creases that have pairwise common tangents. The creases are all circular arcs and alternate mountain and valley.
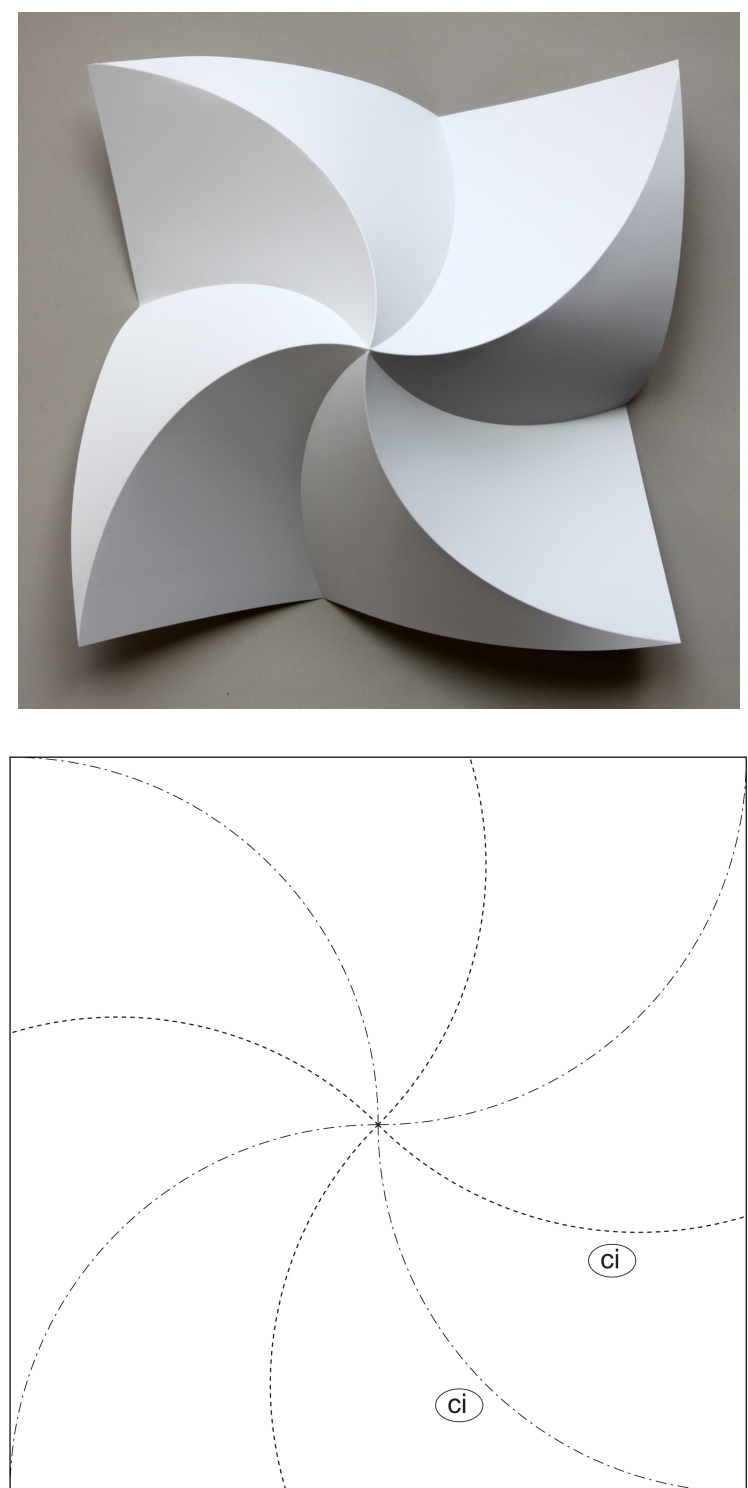

Figure 5. Huffman design using circles (unexploded vertex). 
In Figure 6 we can see a degree- 4 vertex inflated into two degree- 3 vertices. The connecting element in this case is a straight line. The crease pattern uses ellipses that result in more dramatic curvature changes than in Figure 5.
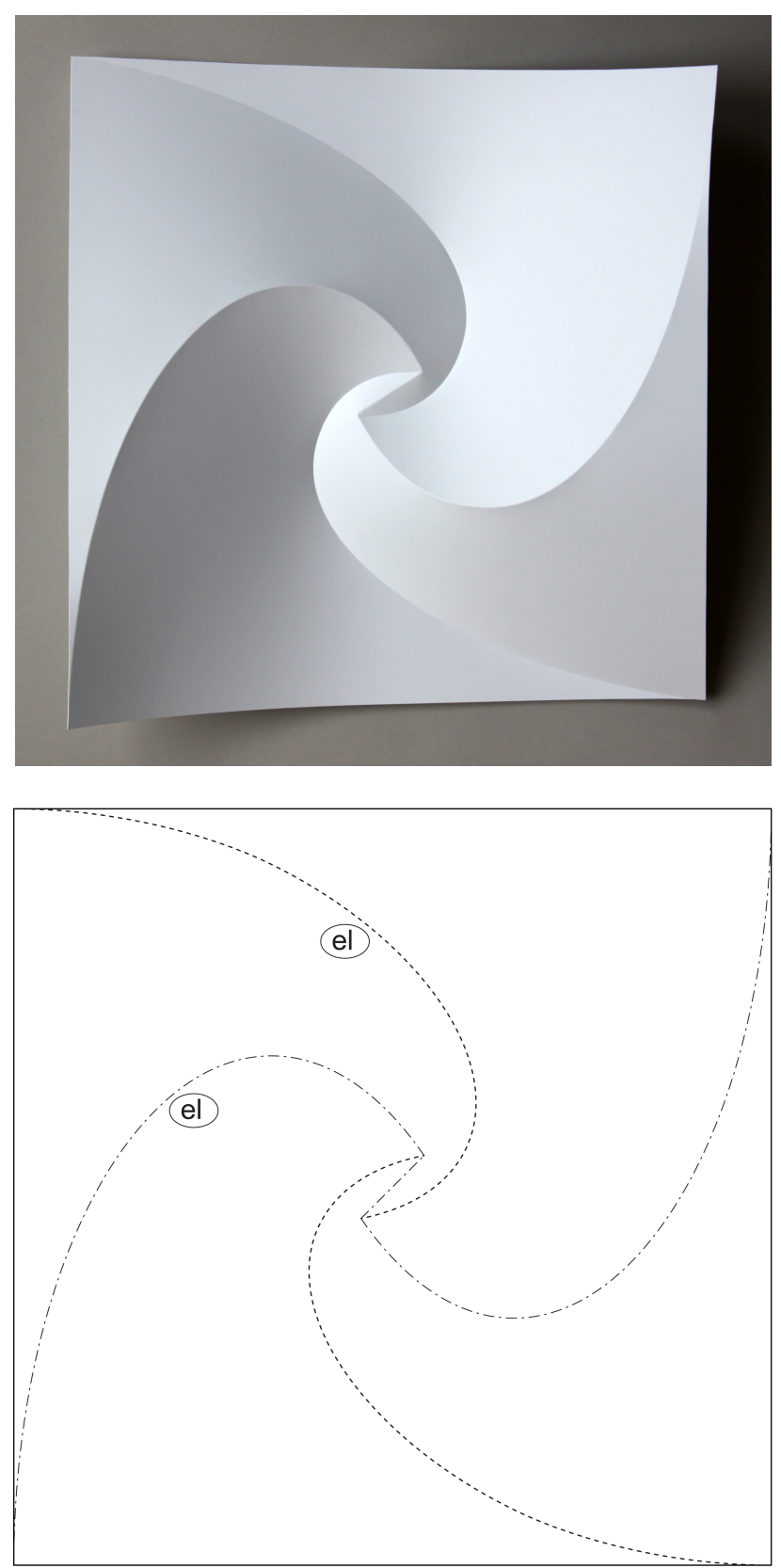

Figure 6. Huffman design using ellipses and a line (exploded vertex). 
In Figure 7 a degree- 4 vertex has been inflated into four degree- 3 vertices. The connecting flat square rotates very little when the design is folded into shape. This example is made of ellipses and is featured on [Haeberli 96].
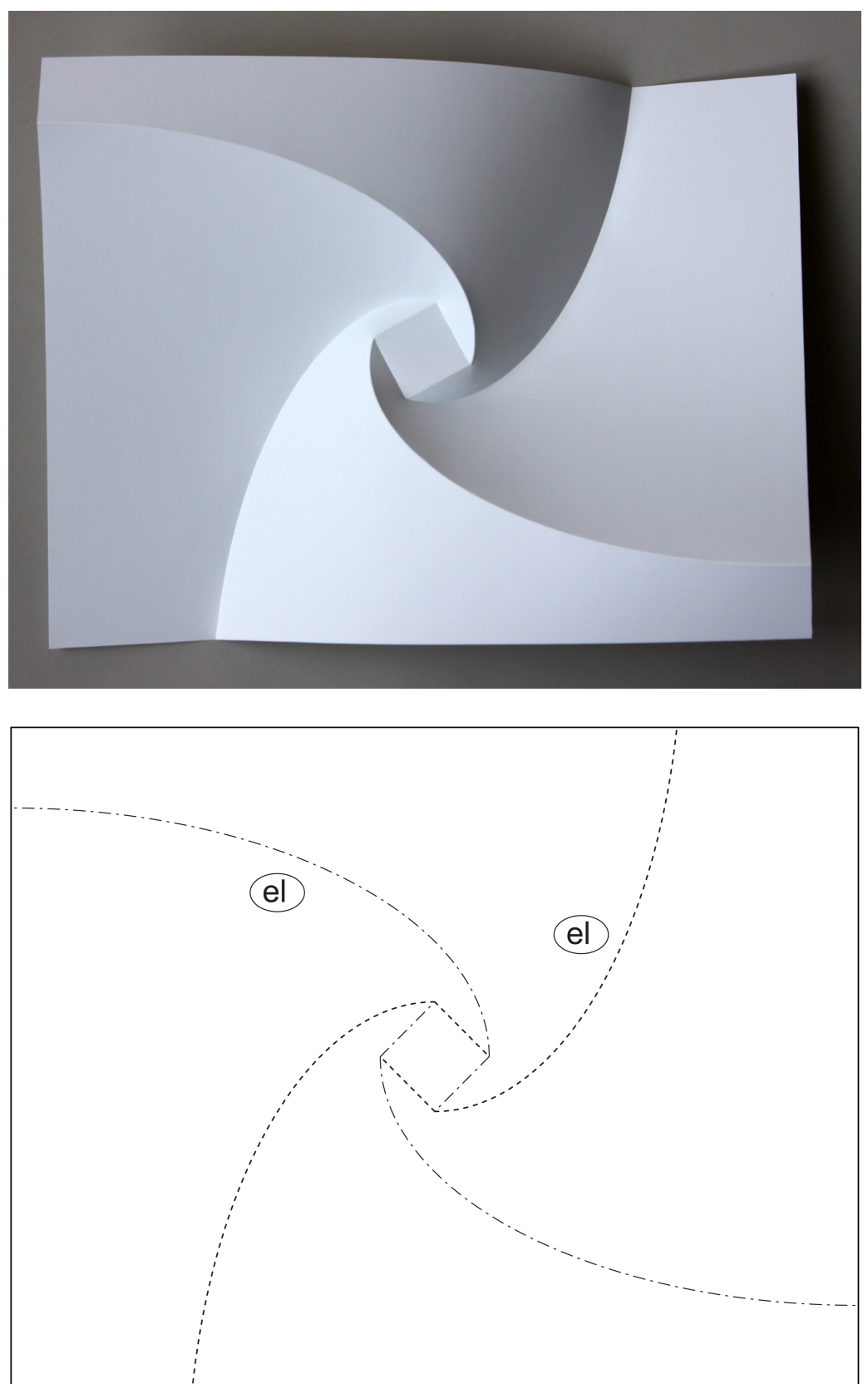

Figure 7. Huffman design using ellipses and a square (exploded vertex). 
Figure 8 shows a crease pattern that displays similar structural properties to socalled "flashers". Simon Guest studied these shapes in terms of how they can curl up into themselves [Guest 92] and Huffman made several of them. This design is made of ellipses that converge in a degree- 6 vertex, which was inflated into six degree- 3 vertices. The connecting element is a flat hexagon that rotates very little in comparison to Guest's shapes and we believe that Huffman was not studying their kinetic behavior, but rather inflated vertices.
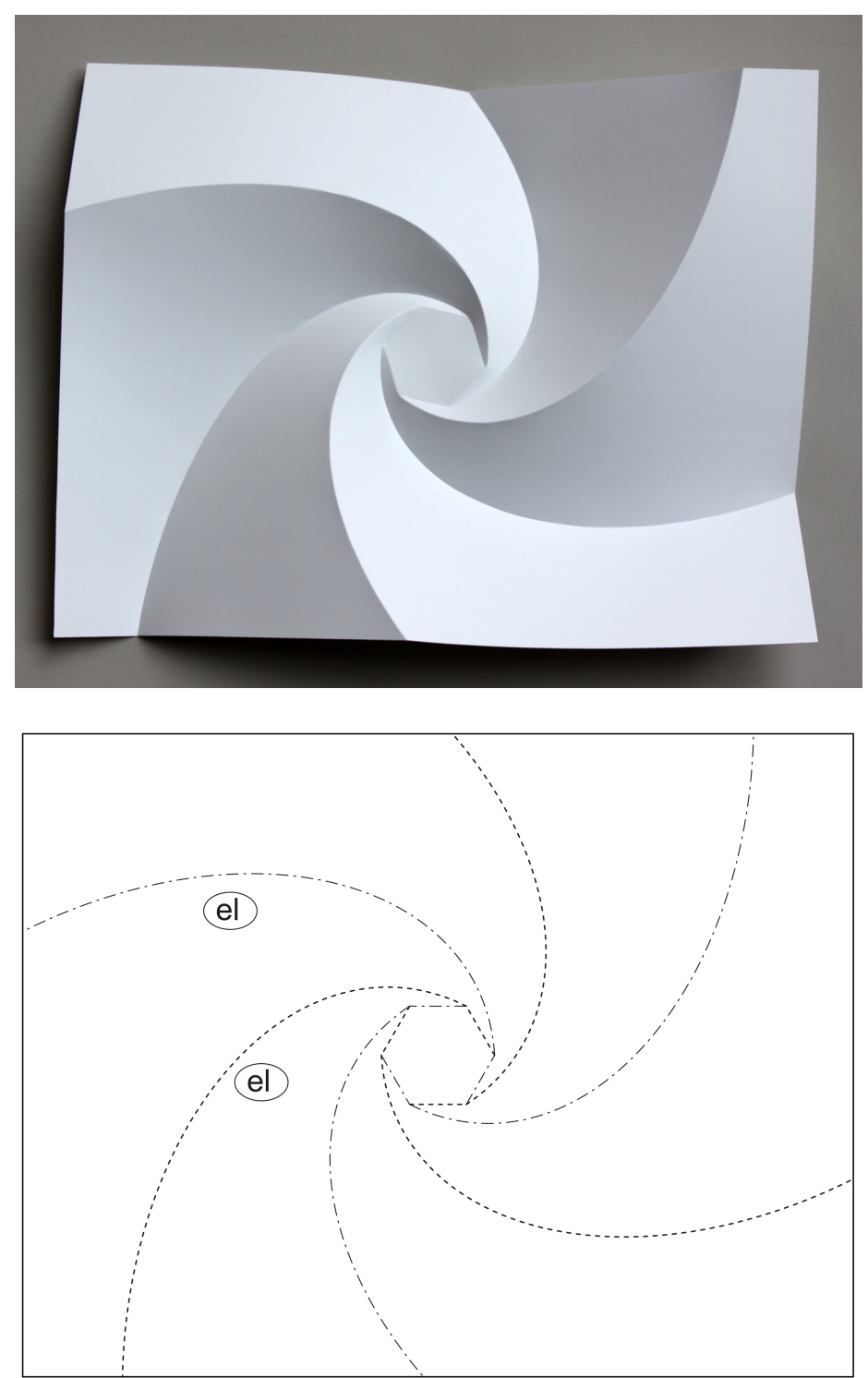

Figure 8. Huffman design using ellipses and a hexagon (exploded vertex). 


\subsection{Tessellations}

Figure 9 shows a tessellation whose tiles are reflectionally and $180^{\circ}$ rotationally symmetric. The crease pattern uses circles and mountain and valley assignments alternate from row to row.
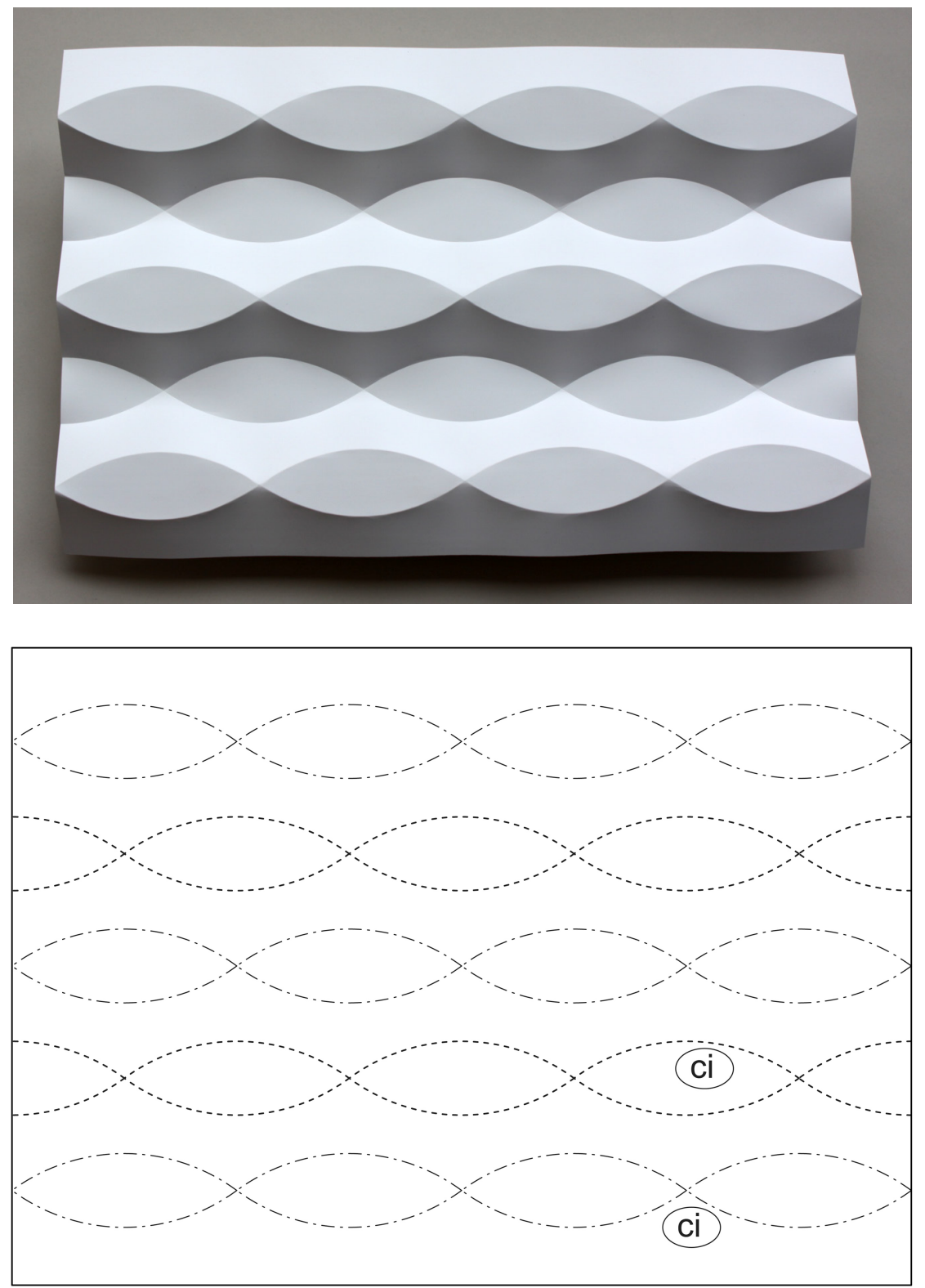

Figure 9. Huffman design using circles. 
Huffman called the tessellation in Figure 10 "Arches" and used parabolas or ellipses which are connected at their focal points. The resulting arches have parallel rulings and the model shown here was made out of paper and uses parabolas.
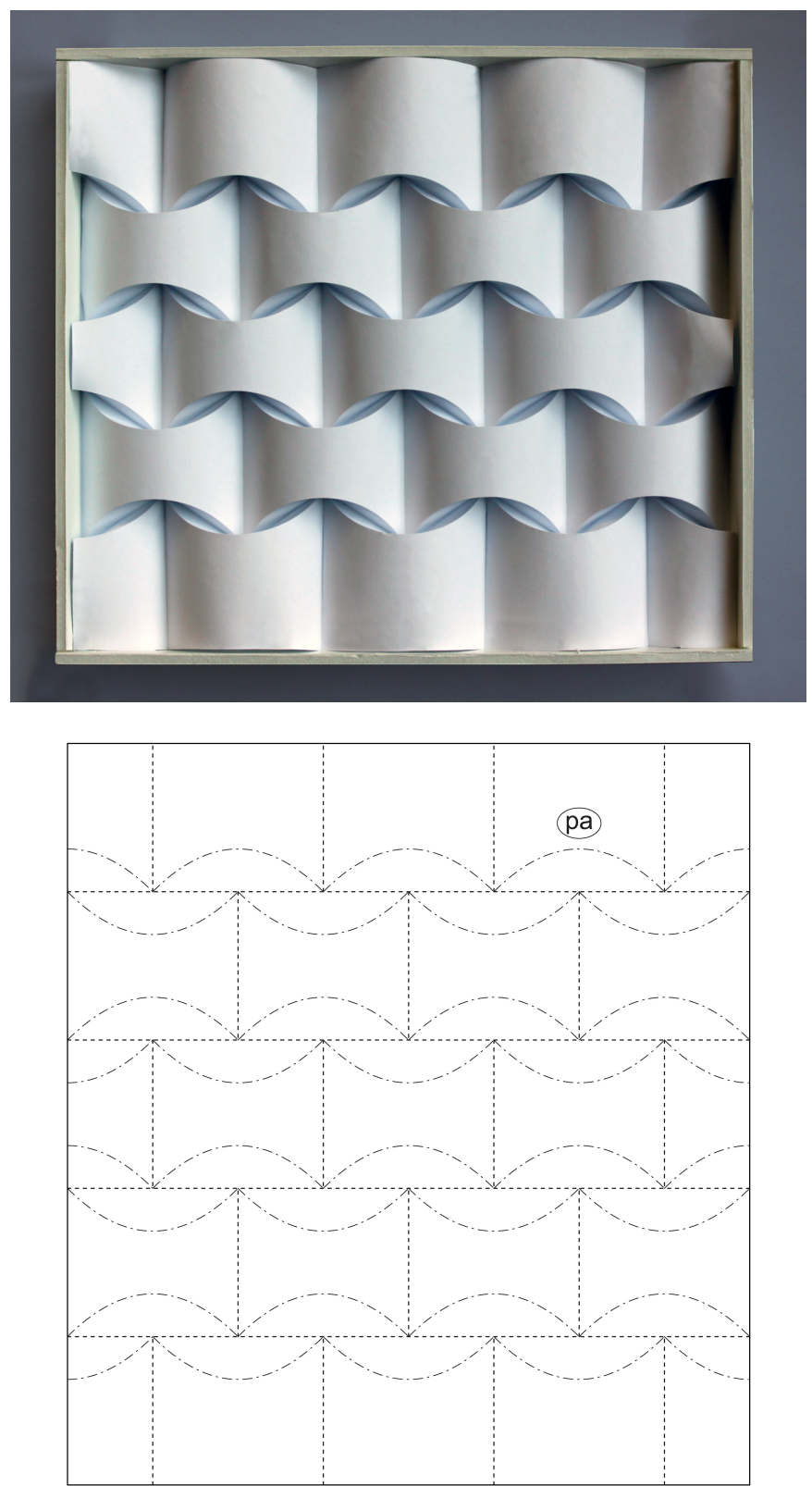

Figure 10. Huffman's “Arches" design using parabolas and lines. 


\subsection{Cones}

Figure 11a shows "Cone reflected 7 times" reconstructed as a digital 3D model. A similar shape was designed by Ron Resch in 1969, which he called "Yellow Folded Cones: Kissing" [Resch 69], where he truncated a cone twice and mirrored it.

Here eight truncated cones are mirrored in an alternating way. The perpendicular cuts to the main axis of the cone result in a circular arc in the crease pattern. The rotated cuts have increasing angles going up from the bottom. The truncated cones need to be unrolled to construct the curves for the crease pattern. These curves are a rare example of Huffman using non-quadratic curves; see Figure $11 \mathrm{~b}$.
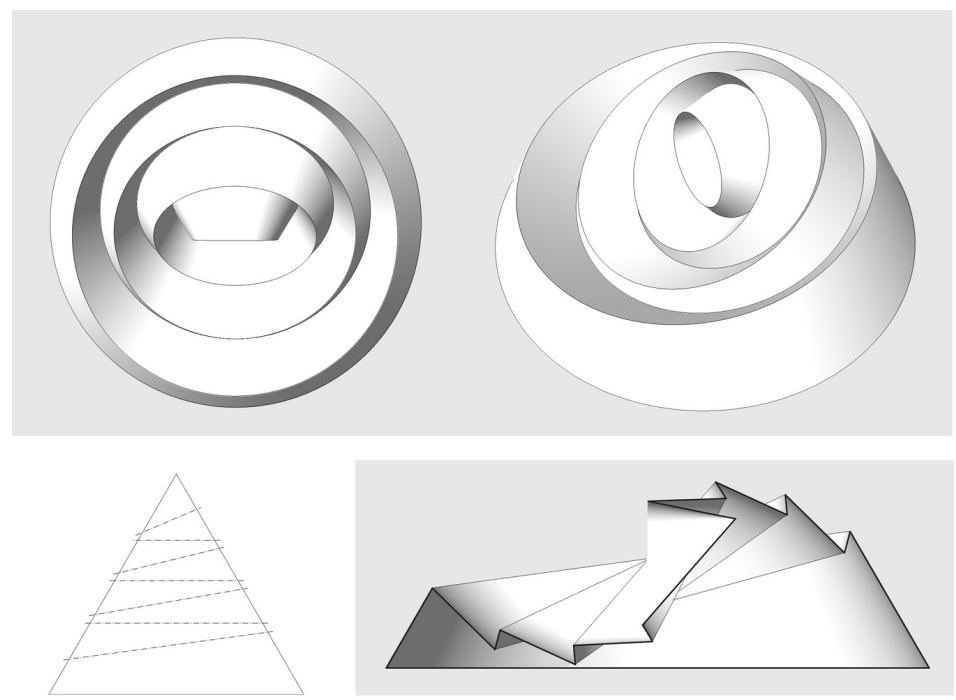

Figure 11a. "Cone reflected 7 times" top and perspective view, section through entire cone and mirrored design

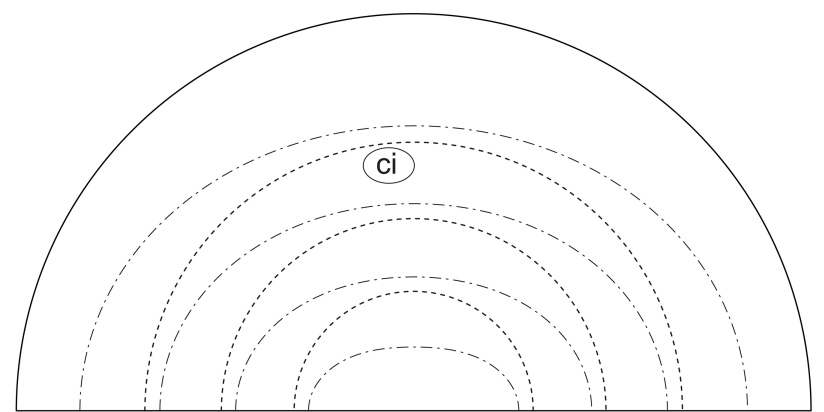

Figure 11b. Crease pattern design using circles and non-quadratic curves. 


\subsection{Complex shapes}

The "hexagonal column with cusps", shown in Figure 12, is remarkable as two sides of the paper meet and create a continuous shape. This stunning and aesthetically very well received example of Huffman's designs has been reconstructed by Saadya Sternberg [SS09] and Robert Lang [Lang 10].

This crease pattern combines partial circles and straight lines. Huffman made many versions of this model with different proportions and sometimes even repeated the entire shape twice in a single crease pattern. Figure 11 shows the crease pattern for two differently proportioned versions. The model shown here was made out of paper.
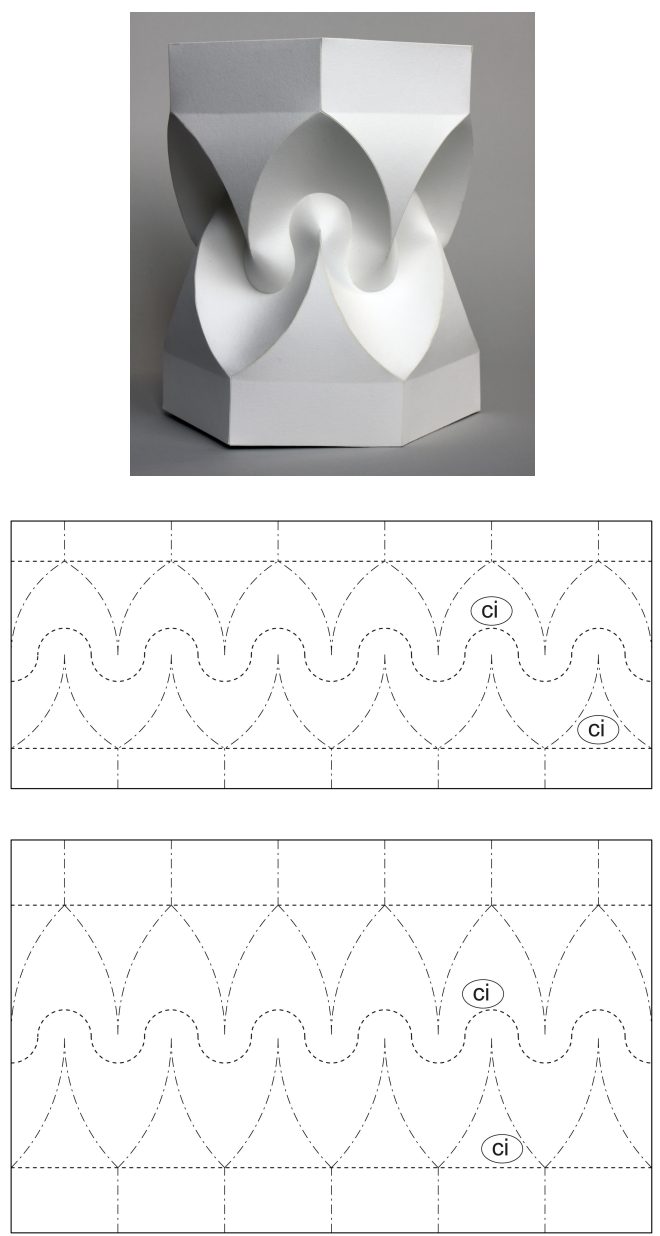

Figure 12. "Hexagonal column with cusps" design using circles and lines The top photograph corresponds to the middle crease pattern. 
The "4-lobed cloverleaf design" is shown in Figure 13. It is symmetric along two axes and is comprised of lines and ellipses. The inner square is folded such that the triangular faces touch one another, a common way to hide material in straight-crease origami, but a rare characteristic among Huffman's designs.
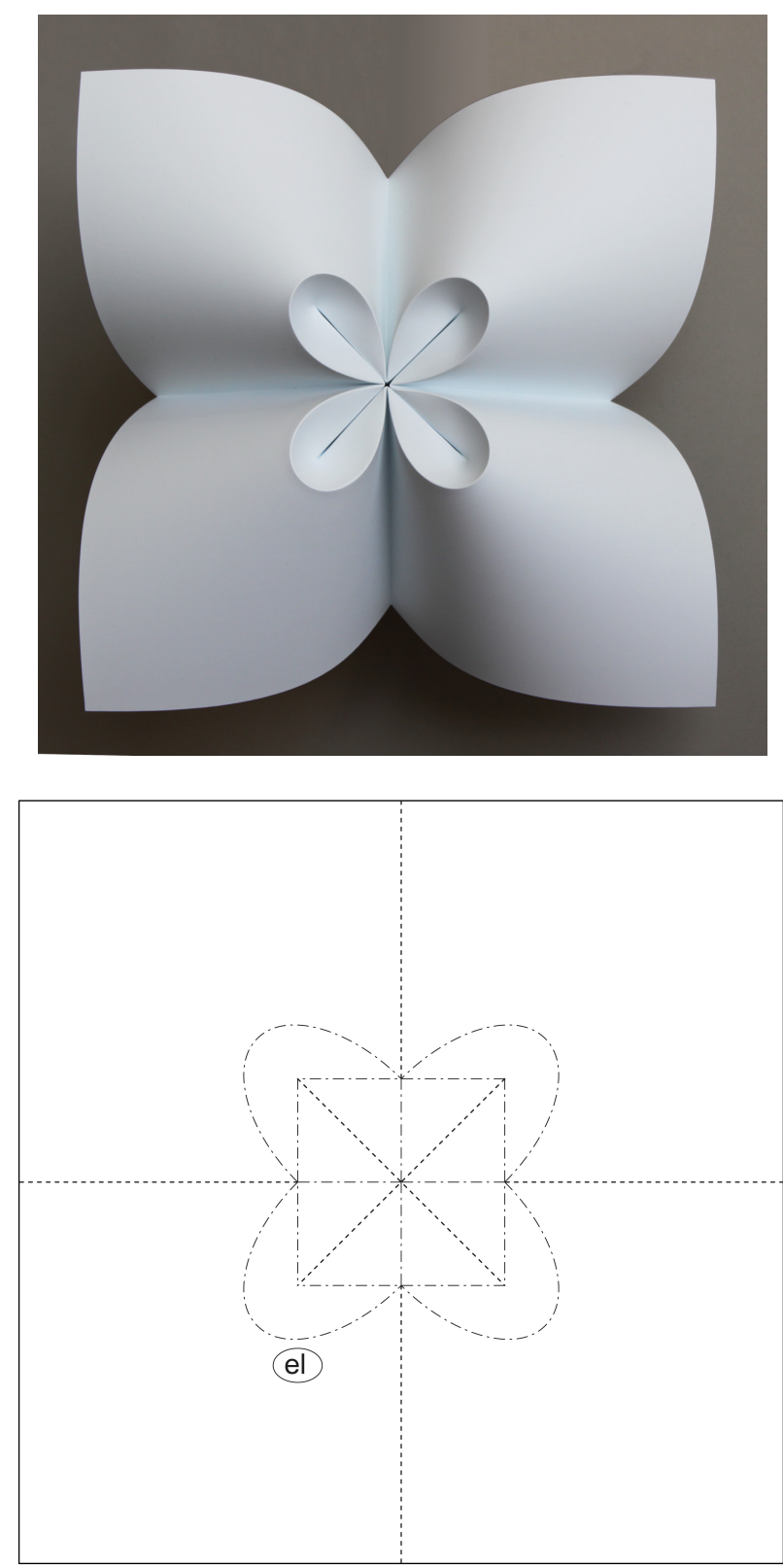

Figure 13. Huffman's "4-lobed cloverleaf design" using ellipses and lines. 
The model in Figure 14, named "One column", was recreated here from white Zanders elephant hide paper." Huffman joined parabolas together to create two wavy extrusions at different scales. This model is particularly striking. It starts with a very simple crease pattern yet creates dramatic features from folding those creases very tightly.
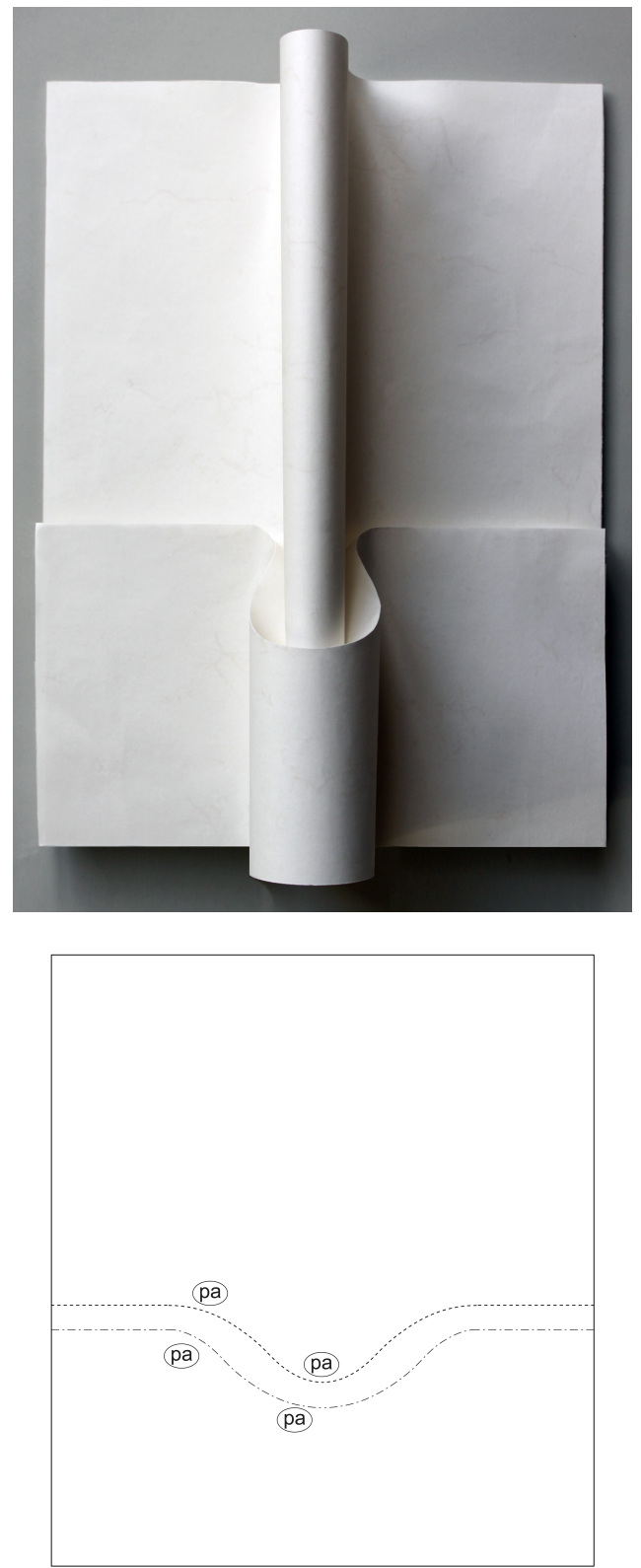

Figure 14. Huffman's “One column” design using parabolas and lines. 


\section{Conclusion}

Our goal is to expose to the world David Huffman's beautiful artwork and the underlying mathematics that he used to create it. We believe that much can be learned from reconstructing and analyzing his final models, which has been the focus of this paper. This reconstruction project is ongoing, with many more models to be studied. We also believe that there is much to be learned from his notes, sketches and working models, a study which is just beginning.

Ultimately we aim to develop a theory for how David Huffman designed his curved-crease foldings, to enable future origami artists and mathematicians to build upon his knowledge and expertise. We regret not being able to develop this theory in direct communication with David, but we are confident that the legacy he left behind will enable a fruitful collaboration.

\section{Acknowledgments}

We thank the Huffman family - in particular, Elise, Linda, and Marilyn Huffman, and Jeff Grubb - for their ongoing collaboration on this project and for their kind hospitality.

Peter Wilson with Makepeace Digital Imaging in Boston, MA has provided us with generous support in making the models. We thank Jenny Ramseyer for her help in making some of the models.

\section{Bibliography}

[Demaine et al 08] Erik D. Demaine and Martin L. Demaine. History of curved origami sculpture, 2008. http://erikdemaine.org/curved/history/

[Fuchs et al 99] Dmitry Fuchs and Serge Tabachnikov. More on paperfolding. The American Mathematical Monthly, 106(1):27-35, January 1999.

[Guest 92] Simon D. Guest and Sergio Pellegrino. Inextensional wrapping of flat membranes. Structural Morphology / Morphologie Structurale, Proceedings of the First International Seminar on Structural Morphology, 203 215, September 1992

[Haeberli 96] Paul Haeberli. Geometric paper folding: Dr. David Huffman. Geometrica Obscura, Nov. 1996. http://www.graficaobscura.com/huffman/ 
[Huffman 76] David A. Huffman. Curvature and creases: A primer on paper. IEEE Transactions on Computers, C-25(10): 1010-1019, Oct.1976.

[Kilian et al 08] Martin Kilian, Simon Flory, Zhonggui Chen, Niloy J. Mitra, Alla Sheffer, and Helmut Pottmann. Curved folding. ACM Transactions on Graphics, 27(3), 75: 1-9, 2008.

[Lang 10] Robert J. Lang. Flapping Birds to Space Telescopes: The Modern Science of Origami. Guest Lecture, MIT, April 26, 2010.

[Ogawa 71] Hiroshi Ogawa. Forms of Paper, New York. Van Nostrand Reingold Company, 1971 (translation of Japanese edition, Tokyo. Orion Press, 1967).

[Resch 69] Ron Resch. Yellow Folded Cones: Kissing (1969-1970),1969-71.

http://www.ronresch.com/gallery/yellow-folded-cones-kissing

[Sternberg 09] Saadya Sternberg. Curves and Flats. Origami ${ }^{4}$ : Proceedings of the 4th International Meeting of Origami. Natick, AK Peters Ltd, Robert J. Lang (editor), 2009.

[Wertheim 04] Margret Wertheim, Cones, Curves, Shells, Towers: He Made Paper Jump to Life, New York Times, June 22, 2004.

[Wingler 69] Hans M. Wingler. Bauhaus: Weimar, Dessau, Berlin, Chicago. MIT Press, 1969. 